\title{
Fatores de risco para consultas em pronto- socorro por crianças asmáticas no Sul do Brasil
}

\section{Asthmatic children's risk factors for emergency room visits, Brazil}

Moema Chatkina, Ana Maria B Menezesa, Elaine Albernaz ${ }^{b}$, Cesar G Victorace Fernando C Barros ${ }^{d}$

aD epartamento de Clínica M édica da U niversidade Federal de Pelotas (U FPel). Pelotas, RS, Brasil. ${ }^{b}$ Departamento M aterno-Infantil da UFPel. 'D epartamento de Medicina Social da UFPel 


\title{
Fatores de risco para consultas em pronto- socorro por crianças asmáticas no Sul do Brasil* \\ Asthmatic children's risk factors for emergency room visits, Brazil
}

\author{
Moema Chatkin a , Ana Maria B Menezesa, Elaine Albernaz ${ }^{\text {b }}$, Cesar G Victora ${ }^{c}$ e \\ Fernando C Barros ${ }^{d}$
}

aD epartamento de Clínica Médica da U niversidade Federal de Pelotas (U FPel). Pelotas, RS, Brasil.

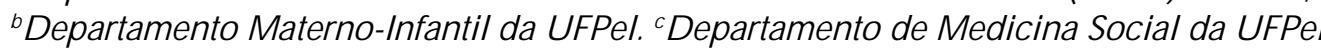

\section{Descritores}

Asma, epidemiologia. Serviços médicos de emergência, utilização\#. Fatores de risco, criança ${ }^{\#}$. Prevalência. Morbidade. Estado asmático.

\section{Keywords}

Asthma, epidemiology. Emergency medical services, utilization ${ }^{\#}$. Risk factors, child". Prevalence. Morbidity. Status asthmaticus.

\section{Resumo}

\section{Objetivos}

Traçar um perfil das crianças asmáticas do município de Pelotas, RS, conhecer o manejo da doença e os fatores de risco associados a consultas em pronto-socorro, motivadas por asma.

\section{Métodos}

Estudo transversal aninhado a um estudo de coorte, realizado na área urbana de Pelotas, cidade de médio porte do Sul do Brasil. Participaram 981 crianças de 4-5 anos, pertencentes à coorte de 1993.

\section{Resultados}

A prevalência de asma encontrada na amostra estudada foi de 25,4\%. A morbidade por asma foi elevada: $31 \%$ das crianças asmáticas haviam procurado o prontosocorro no último ano, $57 \%$ tinham consultado médico e $26 \%$, de 1 a 4 anos, foram internadas por asma. $\mathrm{Na}$ análise geral, escolaridade e renda familiar baixas associaram-se com consultas em pronto-socorro $(\mathrm{RO}=4,1$ para 0 a 4 anos de escolaridade e $\mathrm{RO}=6,5$ para menos de 1 salário-mínimo). Dormir em quartos com 3 ou mais pessoas também mostrou-se associado $(\mathrm{RO}=2,2)$, bem como severidade das crises $(\mathrm{RO}=2,7)$, uso de medicamentos para asma no último ano $(\mathrm{RO}=1,9)$ e internações por asma $(\mathrm{RO}=3,0)$.

Conclusões

A prevalência de asma entre crianças pré-escolares em Pelotas é alta, levando a grande morbidade. Encontraram-se como fatores preditores de consultas em prontosocorro por asma, após análise multivariada, a baixa escolaridade das mães, severidade das crises e internação por asma.

\section{Abstract}

Objectives

To study a sample of asthmatic children to get to know how the disease is managed by caretakers and to identify predictive factors associated with attendance in emergency room for asthma.

\section{Methods}

A cross-sectional study nested in a cohort was undertaken in the urban area of Pelotas, Southern Brazil. 981 children aged 4-5 years, who belong to the cohort of 1993, participated in this study.
*Parte da dissertação de mestrado, apresentada à Faculdade de M edicina da U niversidade Federal de Pelotas, 1999. Recebido em 3/1/2000. Reapresentado em 6/6/2000. Aprovado em 11/7/2000. 


\begin{abstract}
Results
The asthma prevalence in the children sample was $25.4 \%$. Morbidity for asthma was quite high: $31 \%$ of the children were seen in emergency rooms in the last year, $57 \%$ attended medical clinics and $26 \%$ were hospitalized in the first 4 years of life. The crude analysis identified the following predictive factors for emergency room visits: low educational level $(R O=4.1)$, low family income $(R O=6.5), 3$ or more children sleeping in the same room $(R O=2.2)$, severity of asthma attacks $(R O=2.7)$, use of asthma medicines in the last year $(R O=1.9)$ and hospitalizations due to asthma $(R O=3.0)$. Multivariate analyses using logistic regression were used to adjust each variable for the effect of the remainder.

Conclusions

The asthma prevalence among preschool children in Pelotas is high, resulting therefore in high morbidity. The predictor factors for emergency room visits due to asthma found, after multivariate analysis, were mother's low educational level, severity of the asthma attacks and hospitalization.
\end{abstract}

\section{INTRO DU ÇÃO}

A asma é a doença crônica de maior prevalência entre as crianças. ${ }^{8}$ Apesar dos avanços na compreensão da sua fisiopatologia e da crescente oferta de medicações, diversos estudos vêm demonstrando aumento da prevalência, da morbidade e da mortalidade por asma em vários países. ${ }^{2,3}$

A falta de manejo adequado tem sido descrita como uma das principais causas da demanda em salas de emergência por crianças asmáticas. ${ }^{4-6}$ Em estudo realizado na Pensilvânia (USA), em 1994, foi verificado baixo uso de drogas antiinflamatórias para o controle da asma. ${ }^{6}$

A gravidade da doença também tem sido mostrada como um dos fatores responsáveis por consultas em salas de emergência. Estudo sobre acesso aos serviços de saúde no interior de cidades norte-americanas mostrou que as crianças com asma severa raramente usavam medicação preventiva. ${ }^{4}$

Ainda nos Estados Unidos, Halfon et $\mathrm{al}^{7}$ mostrou que crianças pobres e com formas mais severas de asma tiveram menos consultas ambulatoriais do que crianças de melhor nível socioeconômico, apresentando, também, maior probabilidade de receber cuidado médico em serviços de emergência e sendo hospitalizadas $40 \%$ mais vezes e por maior tempo do que crianças asmáticas de melhor nível socioeconômico.

A asma tem um profundo impacto na criança, nas suas famílias e na sociedade. Constata-se um grande número de crianças asmáticas recorrendo aos serviços de emergência, com necessidade freqüente de internações. Os serviços de emergência vêm sendo utilizados como local regular de consultas, o que não é adequado, levando a falhas no tratamento e no con- trole da doença. Como resultante, tem-se um grande ônus, tanto para as crianças asmáticas, com perdas de dias de escola e faltas dos pais ao serviço, como para o sistema de saúde, com custos elevados.

O presente estudo foi realizado com o objetivo de traçar um perfil das crianças asmáticas do município de Pelotas, RS, conhecer o manejo da doença e os fatores de risco associados a consultas por asma em salas de emergência. O presente estudo é um corte transversal de um estudo longitudinal, com a coleta de dados realizada entre novembro de 1997 e abril de 1998.

\section{MÉTO DOS}

Pelotas, onde foi realizado o presente estudo, é uma cidade de médio porte do Sul do Brasil. Participaram 1.363 crianças, com idade entre 4 e 5 anos, que fizeram parte de um estudo longitudinal das crianças nascidas em Pelotas, em 1993, ${ }^{13}$ quando todos os 5.304 nascimentos hospitalares foram recrutados com o objetivo de estudar a influência de fatores sociais e biológicos na saúde, no crescimento e no desenvolvimento infantis. A partir desta coorte, foram realizados quatro acompanhamentos no primeiro ano de vida a subamostras selecionadas de maneira sistemática. Com 1 e 3 meses de idade, 655 crianças foram selecionadas por amostragem sistemática com uma quota fixa semanal. Destas, $649(99,1 \%)$ foram encontradas com um mês, e 644 (98,3\%) aos 3 meses. Para as visitas de 6 e 12 meses de idade, 1.460 crianças foram selecionadas, representando uma amostra sistemática de $20 \%$ de toda a coorte (incluindo as crianças estudadas com 1 e 3 meses), mais todas as crianças nascidas de baixo peso. Destas 1.460 crianças, 1.414 (96,8\%) foram localizadas aos 6 meses, e $1.363(93,4 \%)$ aos 12 meses. O presente estudo tentou localizar as 1.363 crianças que foram localizadas aos 12 meses de idade, compreendendo a amostra sistemática de $20 \%$ dessa 
coorte, o que permitiu estudar exposições de 7,5\% a $9 \%$, com um poder estatístico de $80 \%$ e significância de $5 \%$, detectando um risco relativo de 2 .

Um questionário padronizado pré-codificado foi aplicado às mães das crianças, no período de novembro de 1997 a abril de 1998, por entrevistadores selecionados e treinados para esse fim. As questões sobre sintomas de asma foram baseadas em questionário utilizado pelo estudo multicêntrico internacional de asma e alergia. ${ }^{1}$ Foi utilizado o ponto de corte de 5 pontos nas perguntas sobre sinais e sintomas para o diagnóstico de asma, com base no estudo de Solé. ${ }^{12}$ Nas perguntas, foi utilizado o termo "bronquite" juntamente com "asma" devido à sua ampla utilização na população estudada.

As características biológicas estudadas foram sexo e cor da pele, os fatores socioeconômicos foram avaliados pelas variáveis escolaridade da mãe e do pai, renda familiar em salários-mínimos e classe social Abipeme* (A a E); os fatores ambientais, por meio de perguntas sobre fumo no domicílio; e a aglomeração, pelo número de moradores no domicílio e de pessoas que dormem com a criança. História de eczema ou rinite na criança e asma ou alergia na família, fatores gestacionais como idade da mãe, idade gestacional e fumo durante a gestação, aleitamento materno, baixo peso ao nascer e internação por bronquiolite no primeiro ano de vida, também foram estudados.

Para as crianças que preenchiam os critérios diagnósticos de asma, era investigado o manejo da doença, da morbidade e da gravidade. Os marcadores de gravidade da doença utilizados foram: dificuldade para falar durante a crise, acordar devido ao chiado uma ou mais vezes por semana e número de crises de asma igual ou maior a 12 por ano. Foi considerada como asma grave a criança que apresentou um ou mais marcadores de gravidade.

Cuidado com a criança asmática foi operacionalizado com as variáveis uso e tipo de medicamentos utilizados no último ano, e medidas preventivas tomadas no lar. Quanto à morbidade por asma, investigaram-se as consultas ambulatoriais pela doença, hospitalizações de 1 a 4 anos e consultas ao pronto-socorro, sendo essa última o desfecho analisado.

Para a análise de associação entre cada variável e o desfecho, foi utilizado o teste do qui-quadrado de Pearson e o teste de tendência para associação linear. $\mathrm{O}$ efeito conjunto das variáveis independentes sobre o desfecho foi visto pela regressão logística não condicional, baseada no modelo de análise hierarquizado ${ }^{14}$ (Figura 1), com as variáveis entrando no modelo conforme os níveis hierárquicos. Todas as variáveis foram incluídas na análise multivariada para evitar a perda do efeito de alguma que estivesse sendo confundida negativamente. $\mathrm{O}$ nível de significância de 0,05 foi escolhido para a exclusão ou manutenção das variáveis no modelo.

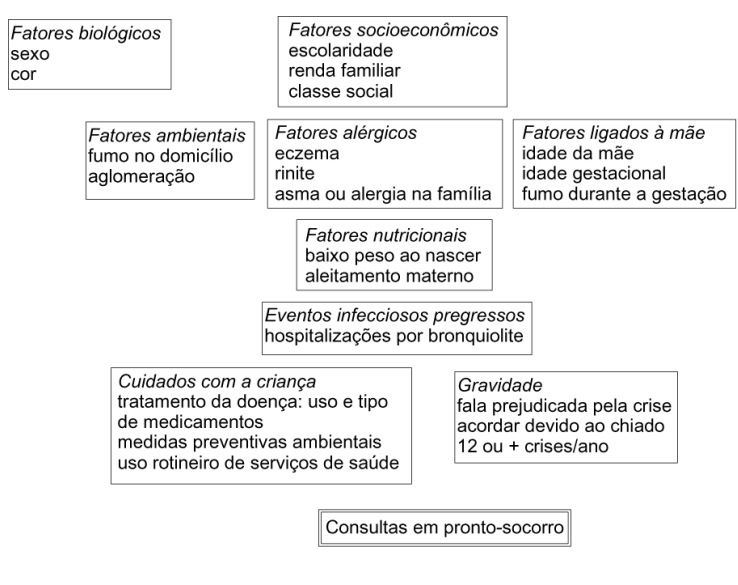

Figura 1- Modelo de análise.

\section{RESU LTAD OS}

Foram estudados $93,4 \%$ da amostra proposta, com um total de $90(6,6 \%)$ perdas e recusas, sendo a mudança de endereço responsável pela maior parte das perdas. A população estudada, após ter sido feita amostragem aleatória das crianças de baixo peso ao nascer, e terem sido retirados os casos excedentes para evitar superamostragem, foi de 981 crianças, correspondendo a $20 \%$ da coorte original.

A prevalência de diagnóstico provável de asma conforme os critérios utilizados (escore 5 ou mais de acordo com os sinais e sintomas apresentados) foi de $25,4 \%$ $(\mathrm{N}=249)$. Um terço de todas as crianças asmáticas haviam procurado serviços de emergência no último ano, $57 \%$ haviam tido consultas ambulatoriais e $26 \%$ haviam sido internadas por asma com idade entre 1 e 4 anos.

As Tabelas 1 e 2 mostram a distribuição das crianças da amostra de acordo com as características individuais e familiares estudadas, comparando as asmáticas com as não asmáticas.

As crianças asmáticas distribuíram-se quase que igualmente entre os dois sexos, não havendo diferença estatisticamente significante com o grupo de crianças não asmáticas. O grupo das crianças com asma apresentou maior proporção de crianças não-brancas 
Tabela 1- Distribuição das crianças de acordo com características individuais. Pelotas, RS, Brasil, 1998.

\begin{tabular}{|c|c|c|}
\hline Variável & Asma - N (\%) & Não-asma - N (\%) \\
\hline \multicolumn{3}{|l|}{ Sexo } \\
\hline $\begin{array}{l}\text { Feminino } \\
\text { Masculino }\end{array}$ & $\begin{array}{l}123(49,4) \\
126(50,6)\end{array}$ & $\begin{array}{l}376(51,4) \\
356(48,6)\end{array}$ \\
\hline \multicolumn{3}{|l|}{ Cor* } \\
\hline $\begin{array}{l}\text { Branca } \\
\text { Não branca }\end{array}$ & $\begin{array}{r}171(68,7) \\
78(31,3)\end{array}$ & $\begin{array}{l}582(79,5) \\
150(20,5)\end{array}$ \\
\hline \multicolumn{3}{|l|}{ Baixo peso ao nascer* } \\
\hline $\begin{array}{l}\text { Não } \\
\text { Sim }\end{array}$ & $\begin{array}{r}214(85,9) \\
35(14,1)\end{array}$ & $\begin{array}{r}676(92,4) \\
56(7,6)\end{array}$ \\
\hline \multicolumn{3}{|l|}{ Idade gestacional } \\
\hline $\begin{array}{l}37 \text { ou mais semanas } \\
<37 \text { semanas }\end{array}$ & $\begin{array}{r}228(91,6) \\
21(8,4)\end{array}$ & $\begin{array}{r}673(91,9) \\
59(8,1)\end{array}$ \\
\hline \multicolumn{3}{|l|}{ Aleitamento materno } \\
\hline$>=9$ meses & $63(25,3)$ & $201(27,6)$ \\
\hline 4 a 8,9 meses & $51(20,5)$ & $164(22,4)$ \\
\hline 1 a 3,9 meses & $88(35,3)$ & $257(35,1)$ \\
\hline \multicolumn{3}{|l|}{ Alergia na criança* } \\
\hline Não & $132(53,0)$ & $525(71,7)$ \\
\hline Sim & $117(47,0)$ & $207(28,3)$ \\
\hline \multicolumn{3}{|l|}{$\begin{array}{l}\text { Hospitalizações por } \\
\text { bronquiolite até } 1 \text { ano }\end{array}$} \\
\hline \multicolumn{3}{|l|}{ bronquiolite até 1 ano } \\
\hline \multirow{3}{*}{ Sim } & $237(95,2)$ & $704(96,6)$ \\
\hline & $12(4,8)$ & $28(3,4)$ \\
\hline & $249100,0)$ & $732(100,0)$ \\
\hline
\end{tabular}

*Teste do qui-quadrado de Pearson $p<0,001$

Tabela 2 - Distribuição das crianças de acordo com características familiares. Pelotas, RS, Brasil, 1998.

\begin{tabular}{|c|c|c|}
\hline Variável & Asma - N (\%) & $\mathrm{N}$ ão-asma - N (\%) \\
\hline $\begin{array}{c}\text { Escolaridade da mãe* } \\
>=9 \text { anos } \\
5-8 \text { anos } \\
0-4 \text { anos }\end{array}$ & $\begin{array}{r}46(18,5) \\
114(45,8) \\
89(35,7)\end{array}$ & $\begin{array}{l}211(28,8) \\
346(47,3) \\
175(23,9)\end{array}$ \\
\hline $\begin{array}{l}\text { Renda familiar* } \\
\text { >10 sm } \\
6,1 \text { a } 10 \mathrm{sm} \\
3,1 \text { a } 6 \mathrm{sm} \\
1,1 \text { a } 3 \mathrm{sm} \\
<1 \mathrm{sm}\end{array}$ & $\begin{array}{r}20(8,0) \\
23(9,2) \\
69(27,7) \\
87(34,9) \\
50(20,1)\end{array}$ & $\begin{array}{r}98(13,4) \\
84(11,5) \\
209(28,6) \\
238(32,5) \\
103(14,1)\end{array}$ \\
\hline $\begin{array}{l}\text { Classe social* } \\
\text { A/B } \\
\text { C } \\
\text { D } \\
\text { E }\end{array}$ & $\begin{array}{l}19(7,6) \\
86(34,5) \\
84(33,7) \\
60(24,1)\end{array}$ & $\begin{array}{l}122(16,7) \\
258(35,3) \\
252(34,4) \\
100(13,4)\end{array}$ \\
\hline $\begin{array}{l}\text { Fumo no domicílio** } \\
\qquad \mathrm{Não} \\
1 \text { a } 10 \text { cigarros/dia } \\
\text { Mais de } 10 \text { cigarros/dia } \\
\text { Fumo durante a gestacão** }\end{array}$ & $\begin{array}{r}105(42,2) \\
87(34,9) \\
57(22,9)\end{array}$ & $\begin{array}{l}379(51,8) \\
232(31,7) \\
121(16,5)\end{array}$ \\
\hline $\begin{array}{l}\text { Fumo durante a gestaçäo** } \\
\text { Não } \\
\text { Sim }\end{array}$ & $\begin{array}{l}148(59,4) \\
101(40,6)\end{array}$ & $\begin{array}{l}509(69,5) \\
223(30,5)\end{array}$ \\
\hline $\begin{array}{c}\text { № moradores no domicílio** } \\
<3 \text { pessoas } \\
>=3 \text { pessoas }\end{array}$ & $\begin{array}{l}125(50,2) \\
124(49,8)\end{array}$ & $\begin{array}{l}424(57,9) \\
308(42,1)\end{array}$ \\
\hline $\begin{array}{l}\text { Dormem com a criança } \\
\text { Nenhuma ou } 1 \text { pessoa } \\
2 \text { pessoas } \\
3 \text { ou mais pessoas }\end{array}$ & $\begin{array}{l}85(34,1) \\
82(32,9) \\
82(32,9)\end{array}$ & $\begin{array}{l}284(38,8) \\
243(33,2) \\
205(28,0)\end{array}$ \\
\hline $\begin{array}{l}\text { Idade da mae no nascımento da criança } \\
20 \text { ou mais } \\
\text { Menos de } 20\end{array}$ & $\begin{array}{r}211(84,7) \\
38(15,3)\end{array}$ & $\begin{array}{l}614(83,9) \\
118(16,1)\end{array}$ \\
\hline $\begin{array}{l}\text { Asma ou alergia na família** } \\
\text { Não } \\
\text { Sim }\end{array}$ & $\begin{array}{r}74(29,7) \\
175(70,3)\end{array}$ & $\begin{array}{l}350(47,8) \\
382(52,2)\end{array}$ \\
\hline & $249(100,0)$ & $732(100,0)$ \\
\hline
\end{tabular}


e o dobro de baixo peso ao nascer. Quase metade dessas crianças tinha história de fenômenos alérgicos como eczema e rinite, em comparação com menos de um terço das crianças sem asma (Tabela 1).

A Tabela 2 mostra que as crianças asmáticas pertenciam a famílias de menor escolaridade e renda familiar que as crianças não asmáticas.

Quanto aos fatores ambientais, também houve diferença estatisticamente significativa entre os dois grupos, com as crianças asmáticas sendo mais expostas a fumo e aglomeração que as não asmáticas.

Em relação às crianças asmáticas, $16,5 \%$ tinham a fala prejudicada durante as crises de asma, $48 \%$ acordavam mais de uma vez na semana devido ao chiado, e 6 tiveram 12 ou mais crises de asma no último ano, resultando em 51,4\% da amostra com marcadores de gravidade para asma. As crianças tiveram em média 3 crises de asma no último ano.

Mais da metade (67\%) das crianças asmáticas usou medicamentos para tratar a asma nos últimos 12 meses, e apenas $34,5 \%$ usaram medicamentos apropriados e tinham menos de 3 fontes de alérgenos no lar.

A Figura 2 mostra os medicamentos usados pelas crianças asmáticas no último ano, indicando que apenas uma criança estava utilizando corticóide inalatório para tratar a doença.

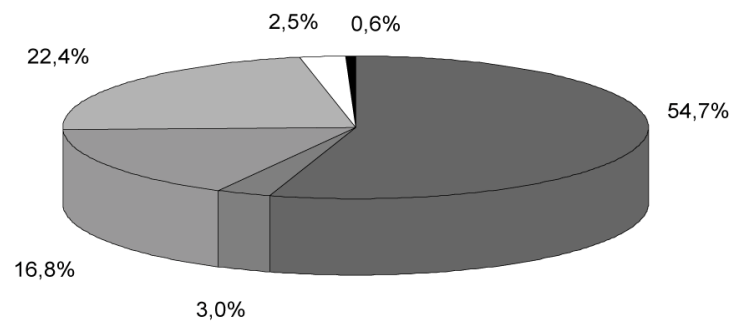

$\begin{array}{lll}\square \text { B2/associado } & \square \text { Xantinas } \\ \square \text { Outros } & \square \text { Cort. vo } & \square \text { Cort. Inalização }\end{array}$

B2/associado: apenas agonistas B2 por qualquer via, ou associados com outro medicamento ou nebulização.

- Outros: descongestionantes sistêmicos, xaropes mucolíticos e antitussígenos.

Figura 2 - Medicamentos usados para asma no último ano pelas crianças asmáticas. Pelotas, RS, 1998.

Na Tabela 3 a renda familiar e a escolaridade baixas mostraram-se associadas a consultas no prontosocorro por asma, sendo que as crianças de famílias de baixa renda familiar e de escolaridade tiveram 4 vezes mais probabilidade de consultar em pronto-socorro por asma do que as crianças de famílias com renda familiar e escolaridade altas.
Entre os fatores ambientais, $\mathrm{o}$ fato de a criança dormir no mesmo quarto com 3 ou mais pessoas levou a uma probabilidade duas vezes maior de procurar serviços de emergência por asma nos últimos doze meses.

A gravidade das crises de asma mostrou-se bastante associada ao desfecho estudado. Entre as crianças que apresentavam crises severas, $41,4 \%$ haviam tido consulta em pronto-socorro, no último ano, com 2,7 vezes mais probabilidade de terem ido à sala de emergência do que as que não tinham crises severas da doença.

As variáveis sobre cuidados com a criança asmática (uso e tipo de medicamentos) estiveram associadas a consultas por asma em pronto-socorro. As crianças que tiveram de usar medicamentos para asma no último ano e as usando agonistas B2 (qualquer via de administração) ou xantinas ou antiinflamatórios inalatórios e a presença de menos de 3 fontes de alérgenos no lar, utilizaram mais o pronto-socorro do que as crianças que não tinham tratamento medicamentoso combinado com medidas preventivas.

Consultas de revisão por asma não se associaram ao desfecho estudado, bem como consultas ambulatoriais pelo mesmo motivo, no último ano. $\mathrm{O}$ fato da criança ter sido internada por asma desde um ano de idade, associou-se a uma probabilidade 3 vezes maior da criança passar por consulta por asma em sala de emergência.

$\mathrm{Na}$ análise multivariada, fatores biológicos não permaneceram no modelo devido à falta de significância estatística. A variável escolaridade materna manteve associação com o desfecho após o ajuste para renda familiar. A variável renda familiar perdeu sua significância, pois, provavelmente, sua associação com o desfecho era decorrente da variável escolaridade que funcionou como fator de confusão positivo, superestimando o verdadeiro efeito da variável renda familiar.

A variável aglomeração perdeu sua significância quando ajustada para escolaridade e fatores alérgicos.

A variável medicamentos apropriados para asma, junto a 3 medidas preventivas no lar, perdeu seu poder explicativo quando ajustada para gravidade. Isso aconteceu porque a variável gravidade era um fator de confusão, ou seja, fazia com que houvesse uma associação que na verdade não existia entre o manejo da doença e o desfecho. O mesmo ocorreu com a variável uso de medicamentos para asma no último ano. A gravidade das crises - após ajustada para escolaridade - e das variáveis do mesmo nível permaneceu no modelo. Do mesmo modo, a internação por asma manteve sua significância. 
Tabela 3 - Distribuição da amostra conforme variáveis biológicas, socioeconômicas e ambientais estudadas e sua associação com "consultas no pronto-socorro por asma no último ano". Pelotas, RS, Brasil, 1998.

\begin{tabular}{|c|c|c|c|}
\hline Variável & $\%$ consultas no PS & OR bruta (IC 95\%) & OR ajust. (IC 95\%) \\
\hline $\begin{array}{c}\text { Renda Familiar } \\
\text { >10 sm } \\
6,1 \text { a } 10 \mathrm{sm} \\
3,1 \text { a } 6 \mathrm{sm} \\
1,1 \text { a } 3 \mathrm{sm} \\
<1 \mathrm{sm}\end{array}$ & $\begin{array}{l}10,0 \\
21,7 \\
29,0 \\
34,5 \\
42,0\end{array}$ & $\begin{array}{l}1,00 \\
2,50(0,43 \text { a } 14,61) \\
3,67(0,78 \text { a } 17,32) \\
4,74(1,03 \text { a } 21,79 * \\
6,52(1,36 \text { a } 31,17)^{*}\end{array}$ & $\begin{array}{l}1,00 \\
1,70(0,28 \text { a } 10,50) \\
2,20(0,43 \text { a } 11,14) \\
2,73(0,55 \text { a } 13,59) \\
3,79(0,73 \text { a } 19,77)\end{array}$ \\
\hline $\begin{array}{l}\text { Escolaridade } \\
\begin{array}{l}>=9 \text { anos } \\
5-8 \text { anos } \\
0-4 \text { anos }\end{array}\end{array}$ & $\begin{array}{l}13,0 \\
33,3 \\
38,2\end{array}$ & $\begin{array}{l}1,00 \\
3,33(1,30 \text { a } 8,55)^{* *} \\
4,12(1,58 \text { a } 10,75)^{* *}\end{array}$ & $\begin{array}{l}1,00 \\
2,77(1,03 \text { a } 7,42)^{*} \\
2,92(1,06 \text { a } 8,07)^{*}\end{array}$ \\
\hline $\begin{array}{l}\text { Dormem com a criança } \\
\text { N enhuma ou uma pessoa } \\
2 \text { pessoas } \\
3 \text { ou mais pessoas }\end{array}$ & $\begin{array}{l}24,7 \\
28,1 \\
41,5\end{array}$ & $\begin{array}{l}1,00 \\
1,19(0,60 \text { a } 2,37) \\
2,16(1,12 \text { a } 4,18)^{*}\end{array}$ & $\begin{array}{l}1,00 \\
0,95(0,46 \text { a } 1,96) \\
1,77(0,88 \text { a } 3,58)\end{array}$ \\
\hline $\begin{array}{l}\text { Não } \\
\text { Sim }\end{array}$ & $\begin{array}{l}20,7 \\
41,4\end{array}$ & $\begin{array}{l}1,00 \\
2,71(1,54 \text { a } 4,77)^{* *}\end{array}$ & $\begin{array}{l}1,00 \\
1,99(1,03 \text { a } 3,84)^{*}\end{array}$ \\
\hline $\begin{array}{l}\text { U so de medicamentos } \\
\text { Não } \\
\text { Sim }\end{array}$ & $\begin{array}{l}18,3 \\
37,7\end{array}$ & $\begin{array}{l}1,00 \\
2,71(1,42 \text { a } 5,14)^{* *}\end{array}$ & $\begin{array}{l}1,00 \\
1,99(0,90 \text { a } 4,40)\end{array}$ \\
\hline $\begin{array}{l}\text { Tipo de medicamento } \\
\text { O utros } \\
\text { Adequados para asma } \\
\text { Internacões por asma }\end{array}$ & $\begin{array}{l}26,4 \\
40,7\end{array}$ & $\begin{array}{l}1,00 \\
1,91(1,10 \text { a } 3,33)^{*}\end{array}$ & $\begin{array}{l}1,00 \\
0,94(0,47 \text { a } 1,88)\end{array}$ \\
\hline $\begin{array}{l}\text { Internações por asma } \\
\text { Nẫo } \\
\text { Sim }\end{array}$ & $\begin{array}{l}24,9 \\
50,0\end{array}$ & $\begin{array}{l}1,00 \\
3,02(1,67 \text { a } 5,47)^{* *}\end{array}$ & $\begin{array}{l}1,00 \\
2,71(1,42 \text { a } 5,16)^{* *}\end{array}$ \\
\hline
\end{tabular}

$* \mathrm{p}<0,05$

$* * p<0,001$

PS - Pronto-socorro

OR - "O dds ratio"

IC - Intervalo de confiança

Permaneceram no modelo como fatores preditores de "consultas ao pronto-socorro por asma no último ano" a baixa escolaridade das mães, a gravidade das crises de asma e a internação por asma de crianças com 1 a 4 anos, após o ajuste para todas as variáveis do modelo.

\section{DISCU SSÃO}

O presente estudo apresentou algumas limitações, como a causalidade reversa, viés que pode afetar estudos transversais decorrente do fato de as informações sobre a doença e os fatores de determinação serem coletados simultaneamente. Os fatores prognósticos identificados, baixa escolaridade das mães e internações por asma de crianças com 1 a 4 anos, certamente já estavam presentes antes da ocorrência do desfecho, isto é, consultas em pronto-socorro por asma nos últimos doze meses. Pode-se, portanto, afirmar que nesse caso não há presença do viés. $\mathrm{O}$ fator prognóstico gravidade poderia ser decorrente da falta do controle adequado da doença pelo atendimento em salas de emergência e não em ambulatórios ou serviços especializados. Mesmo assim, a identificação desse fator é importante, pois crianças que apresentam marcadores de gravidade têm risco de apresentar crise grave ou estão sendo mal manejadas clinicamente.

Possíveis aspectos como o de memória podem ocorrer quando o desfecho ou o fator estudado não são atu- ais e o entrevistado não tem condições de lembrar o fato. Com o objetivo de minimizar essa limitação, as perguntas foram limitadas ao último ano e acredita-se que, sendo a asma uma doença que causa ansiedade na mãe e na criança, a mesma deve ser bem recordada.

A prevalência de asma na população estudada foi elevada, sendo que uma de cada 4 crianças pré-escolares apresentaram asma de acordo com o escore utilizado. Não existem outros estudos de prevalência de asma na população estudada, impedindo a comparação e o conhecimento de tendência temporal da ocorrência da doença.

Utilizando o mesmo escore, Solé et al ${ }^{12}$ encontraram uma prevalência de 23,8\% em meninos e 20,4\% nas meninas de 6-7 anos em São Paulo.

As crianças asmáticas distribuíram-se quase igualmente entre os dois sexos, diferindo de dados da literatura que apontam para uma maior ocorrência de asma entre os meninos. ${ }^{10}$ Baixo peso ao nascer, cor não-branca e história de fenômenos alérgicos estiveram associados à asma. Esses fatores têm sido descritos como de risco para a doença em diversos estudos. ${ }^{10}$

Quanto ao manejo da doença, os agonistas B2 foram os medicamentos mais utilizados pelas crianças asmáticas, sendo que apenas uma delas estava usando antiinflamatório por via inalatória e, para cerca de 
$22 \%$, haviam sido prescritas drogas não indicadas para o tratamento da doença, como antitussígenos e antiinflamatórios não-esferoidais. Isso aponta para algumas hipóteses: manejo inadequado da doença de acordo com os consensos nacionais e internacionais para o manejo da asma, ${ }^{9,11}$ dificuldades de acesso a cuidados médicos por essas crianças, ou falta de conhecimento da doença por parte das famílias.

Deficiências na qualidade do cuidado médico para asma foram relatadas por Crain et al, ${ }^{4}$ estudando o acesso a tratamento de qualidade para crianças com asma no interior de grandes cidades nos Estados Unidos, de acordo com indicadores contidos no Manual para Diagnóstico e Manejo da Asma. ${ }^{9}$ Friday et al ${ }^{6}$ também encontraram falta de manejo adequado em crianças que consultavam serviços de emergência para asma. Agonistas B2 foram identificados como os medicamentos mais utilizados e apenas $16 \%$ das crianças usavam corticoesteróides inalatórios, conforme os resultados observados por Friday et al. ${ }^{6}$

Pelo fato de não haver medidas de função pulmonar, a gravidade da doença foi avaliada por "marcadores de gravidade" - 12 ou mais crises por ano, acordar devido ao chiado e fala prejudicada durante a crise. Observou-se que, apesar de cerca de metade das crianças apresentarem gravidade, apenas um terço das mesmas utilizaram medicamentos para asma e tinham medidas preventivas no lar. Isso reforça a idéia da falta de um manejo adequado da asma em nosso meio.

Gravidade foi um fator-prognóstico de consultas em pronto-socorro por asma, assim como baixa escolaridade das mães e internações por asma. Crain et al, ${ }^{4}$ estudando o acesso a tratamento adequado para asma, viram que as crianças com asma grave tinham maior probabilidade de usar salas de emergência do que aquelas com asma leve. Farber et al, ${ }^{5}$ pesquisando o perfil de crianças que visitavam salas de emergência por asma, observaram que a maioria delas tinha gravidade e era acompanhada por adultos com baixa escolaridade. $\mathrm{O}$ presente estudo mostrou que as crian- ças com asma grave eram as que também utilizavam medicamentos apropriados para asma e tinham 3 medidas preventivas no lar, mas esse manejo da doença provavelmente era inadequado devido à subutilização de medicação preventiva. Assim, essas crianças, que deveriam ter proteção em relação ao desfecho, mostravam maior risco e a variável, quando ajustada para gravidade, não permaneceu como fator preditor ou protetor de consultas ao pronto-socorro por asma. Certamente, o grande número de crianças com indicadores de gravidade encontrado no presente estudo está associado à falta de manejo adequado da doença.

Admissões ao hospital foram também fatores preditores de visitas a salas de emergência em outros estudos. ${ }^{5,6,15}$ Wasilewski et al ${ }^{15}$ observaram que crianças com hospitalização prévia por asma apresentavam alto risco de utilizar serviços de emergência. Sua conclusão foi que famílias usando freqüentemente serviços de emergência necessitam de educação sobre asma.

Friday et al, ${ }^{6}$ procurando identificar fatores antecedentes em pacientes que consultavam serviços de emergência por sibilância, observou que existe um grupo de pacientes com sibilância recorrente, com manejo inadequado, o que acarreta hospitalizações de maior custo.

Conclui-se que a prevalência de asma entre crianças pré-escolares em Pelotas é alta. As crianças asmáticas não diferem quanto ao sexo, são predominantemente de famílias de baixa renda e de baixa escolaridade, expostas a fumo e aglomeração. Metade delas apresenta gravidade nas crises e não tem manejo adequado da doença, o que acarreta uma elevada utilização dos serviços de saúde que parece não estar sendo resolutiva.

Recomenda-se a implantação de um Programa de Controle para Asma que contemple ações educativas e preventivas, visando a um manejo adequado da doença, diminuindo assim a sua morbimortalidade.

\section{REFERÊN CIAS}

1. Asher MI, Keil U, Anderson HR, Beasley R, Crane J, Martinez F et al. International Study of Asthma and Allergies in Childhood (ISAAC): rationale and methods. Eur Respir J 1995;8:483-91.

2. Burney PG, Chinn S, Rona RJ. Has the prevalence of asthma increased in children? Evidence from the national study of health and growth 1973-86. BMJ 1990;300:1306-10.
3. Chatkin JM, Barreto SSM, Fonseca N, Gutiérrez CA, Sears MR. Trends in asthma mortality in young people in southern Brazil. Ann Allergy Asthma Immunol 1998;82:287-92.

4. Crain EF, Kercsmar C, Weiss KB, Mitchell H, Lynn H Reported difficulties in acess to quality care for children with asthma in the inner-city. Arch Pediatr Adolesc Med 1998;152:333-9. 
5. Farber HJ, Johnson C, Beckerman RC. Young inner-city visiting the emergency room (er) for asthma: risk factors and chronic care behaviors. J Asthma 1998;35:547-52.

6. Friday $G A$, Khine $H$, Lin $M$, Caliguiri LA. Profile of children requiring emergency treatment for asthma. Ann Allergy Asthma Immunol 1997;78:221-4.

7. Halfon N, N ewacheck PW. Childhood asthma and poverty: differential impacts and utilization of health services. Pediatrics 1993;91:56-61.

8. M C Fadden JR, Gilberte IA. Asthma. N Engl J Med 1992;327:1928-37.

9. National Asthma Education and Prevention Program. Guidelines for the diagnosis and management of asthma. $2^{\text {nd }}$. Expert Panel Report: Bethesda; 1997.

10. Schwartz J, Gold D, Dockery DW, Weiss ST, Speizer FE. Predictors of asthma and persistent wheeze in a national sample of children in the U nited States: association with social class, perinatal events and race. Am Rev Respir Dis 1990;142:555-62.
11. Sociedades Brasileiras de Alergia e Imonupatologia, Pediatria e Pneumologia e Tisiologia. $2^{\circ}$ Consenso Brasileiro no Manejo da Asma, 1998. J Pneumol 1998;24:171-276.

12. Solé D, Yamasa E, Vana AT, Costa-Carvalho BT, Naspit CK. Prevalence of asthma and related symptoms in school-age children in São Paulo, Brazil: international study of asthma and allergies in children. J Asthma 1999;36:205-12.

13. Victora CG, Barros FC, Halpern R, Menezes AM, Horta $B L$, Tomasi $E$ et al. Estudo longitudinal da população materno-infantil da região urbana do Sul do Brasil, 1993: aspectos metodológicos e resultados preliminares. Rev Saúde Pública 1996;30:34-5.

14. Victora CG, Huttly SR, Fuchs SC, O linto MTA. The role of conceptual frameworks in epidemiological analysis: a hierarchical approach. Int J Epidemiol 1997;26:224-7.

15. Wasilewski Y, Clarck N M, Levison MJ, Levin B, Mellins RB. Factors associated with emergency department visits by children with asthma: implications for health education. Am J Public Health 1996;86:1410-5. 